\title{
Controlled fading of soluble sulphur dyed cotton fabric by oxidation discharge printing
}

\begin{abstract}
The quest of this study is to accomplish a good fading effect on cotton fabric by discharge printing method. Cotton woven fabrics were padded with innovative sulphur dyes in solubilized form (without affinity) in blue colour and fixative was applied on them to impart fastness properties. Faded patterns were produced on them by discharge printing techniques by using pastes containing potassium permanganate, sodium perborate and benzoyl peroxide plus sodium chlorate as oxidizing discharge agents. Hectorite was used as thickener for printing with each oxidant. The effects were similar to wash down effect on denim materials but they were controlled and conformed to design on the screen. Variation in the degree of fading with concentration of oxidizing agent and thickener was also investigated. Fading increased with increase in concentration of the oxidizing agents. In case of benzoyl peroxide, its combination with hectorite was the best and slightly lower fading was found in case of potassium permanganate with hectorite. Fading was found to be dependent on the colour of the dye also. The effects of fading by all the three oxidizing agents on the strength of the fabrics were also studied. There was a loss of strength in all cases. The reduction in strength correlated with degree of fading (greater DE). Higher the value of DE, bigger was the loss in strength. Rubbing fastness of faded and unfaded samples was assessed. Faded specimens displayed higher fastness than the unfaded ones.
\end{abstract}

Keywords: cotton, fading, discharge printing, thickener
Volume 4 Issue 3 - 2018

\author{
Mohammad Mahbubul Alam,' Koushic \\ Uddin,' Ershad Khan,' Nisharat Tanzeem, ${ }^{2}$ \\ Imtiaz Uddin Khondakar, ${ }^{2}$ Mustafizur \\ Rahman $^{3}$ \\ 'Assistant Professor, Ahsanullah University of Science and \\ Technology, Bangladesh \\ ${ }^{2}$ Bachelor Students, Ahsanullah University of Science and \\ Technology, Bangladesh \\ ${ }^{3}$ Professor, Ahsanullah University of Science and Technology, \\ Bangladesh
}

\author{
Correspondence: Mohammad Mahbubul Alam, Assistant \\ Professor, Ahsanullah University of Science and Technology, \\ Dhaka, Bangladesh, Tel 01914712001, \\ Email mahbubula@gmail.com
}

Received: February 19, 2018 | Published: May 08, 2018

\section{Introduction}

Nowadays, vintage look denim is not only the main fashion attraction to the youth but also to the elder people. Faded appearance in denim garment is achieved through numerous physical and chemical finishing. But all the fading methods possess a common drawback which is the controlling the precise fading as well as its reproducibility. This being a blind process, considerable amount of skill and experience is essential. So, the unacceptable effects and hence rejects are not always avoidable. That is why controlled and reproducible fading of denim garment has become a necessity. Discharge printing is a very common printing style which has been practiced for a long period of time to produce localized fading.

The use of oxidizing agents such as sodium hypochlorite, bleaching powder and potassium permanganate is quite common to produce various wash effects by treating cotton readymade garments from sulphur/indigo dye in a wash wheel in a special manner. Sodium formaldehyde sulfoxylate has been used on a commercial scale as a reductive agent in discharge printing. However, it is quite toxic and produces formaldehyde, a known human carcinogen associated with nasal sinus cancer and nasopharyngeal cancer.

Besides faded blue jeans, faded garment with wide ranges of shades are the new fashion demand. To meet this new fashion demand, Archorma has recently introduced a new generation sulphur dye with wide shade gamut branded as Optisul which is claimed to fulfill the requirements of international standard eco-certifications as well as RSL's of major brands and retailers regarding hazardous chemicals and environmental impact. This dye is especially suitable for wash down looks. Here, in this current study fabrics dyed with these dyes (blue and brown) are faded with the discharge printing method using three different oxidizing agents (potassium permanganate, sodium perborate and benzoyl peroxide) with varying concentrations as well as thickener (hectorite).

\section{What are sulphur dyes?}

Sulphur dye is defined as a dye, containing sulphur both as an integral part of the chromophore and in attached polysulphide chains, normally applied in the alkali-soluble reduced (leuco) from a sodium sulphide solution and subsequently oxidized to the insoluble form in the fibre. ${ }^{1}$ Sulphur dyes possess sulphur linkages $(\mathrm{S}-\mathrm{S})$ and are insoluble in water. These are converted to thiols $(-\mathrm{S}-\mathrm{H}-)$ through reduction and then to sodium salt $(-\mathrm{S}-\mathrm{Na}-)$ with alkali; the reduced and solubilized dye shows affinity for cellulose. After dyeing, dyed cotton is oxidized to restore original dye structure. Physical interaction between dye and cotton occurs (Figure 1).

$$
\begin{gathered}
\text { Reduction } \\
\text { Dye-S-S-Dye } \rightarrow \text { Dye-SH-HS-Dye } \rightarrow \text { Dye-SNa } \rightarrow \text { NaS-Dye } \rightarrow \\
\text { Dye-SH-HS-Dye }
\end{gathered} \rightarrow
$$

Figure I Dyeing of cellulose with sulphur colour.2 


\section{Solubilized sulphur dye}

The water-soluble sulphur dyes dissolve in water and they can be dyed with sodium sulphide and soda ash. They are easy to handle, contain less insoluble matters making them suitable for package dyeing and possess superior levelling properties. There are various ways of making stabilized dye. The sulphur dyes solubilized by thiosulphonic acid are prepared by the action of sodium sulphite or bisulphite on the sulphur dye. Solubilized sulphur dyes have some unique features like as:

a. Good combinability of all elements

b. Fixation without the need of polymerization treatment

c. Especially suitable for wash down looks

d. Easy to clean equipment after dyeing

e. Possibility of reusing the dyeing bath

f. Suitable for mechanical, chemical and modern wash-down technologies (laser, ozone, etc.). ${ }^{3}$

\section{Discharge printing}

In discharge printing, the fabric is first dyed to a solid shade and then printed with a paste containing chemicals capable of destroying the colour during the following steaming process. This allows printing of white designs on a ground colour of any depth with a pattern definition that is much higher than would be possible by direct colour printing. If the paste contains dyes resistant to the discharging agent, these dyes, called illuminating colours, will colour the printed areas. ${ }^{4}$

An early and, one might say, classical example is the discharge printing of cotton dyed with indigo, the characteristic colour of which can be destroyed either by oxidation or reduction (Figure 2).

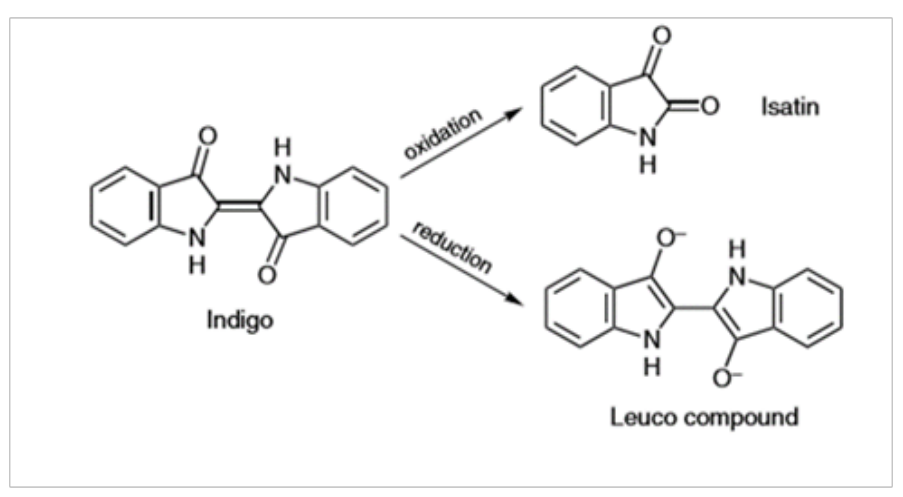

Figure 2 Colour destroyed either by oxidation or reduction.

An oxidizing agent, such as a thickened $10 \%$ solution of sodium dichromate, is printed on to the indigo-dyed material and dried. When this is passed through a hot bath of sulphuric and oxalic acid, chromic acid is liberated and the indigo in the printed areas is oxidized to isatin. The oxalic acid reduces any excess chromic acid which might be formed and which would otherwise gradually discharge the ground colour. Since isatin is soluble in alkali, the final step is to run the fabric through an alkali bath - in an open soaper, for example - where upon the isatin is removed to give a white discharge pattern. The main problem with this process is the adverse effect of the oxidizing agent and the acids on the cotton substrate, which can easily be tendered under this conditions. ${ }^{5}$

\section{Hectorite}

Hectorite clay is generated from soils and sedimentary deposits through weathering, diagenesis and hydrothermal effects, the chemical composition of hectorite included: sodium, lithium, magnesium, silicon, hydrogen and oxygen. This process involves degradation and transformation of precursor phyllosilicates, including precipitation from solution. Thus, clays are derived from various abundant minerals in soils, influenced by conditions that contain basic igneous rocks and also soil clay fractions from arid climates, originating from granitic pediments. Some hectorite samples can be found naturally in the Amazon region. However, this hectorite occurrence is rare due to the fact that the Amazon climate is normally warm humid, causing acidic lixiviation in soils, conditions that are unfavorable for lamellar structure formation (Figure 3) (Figure 4). ${ }^{6}$

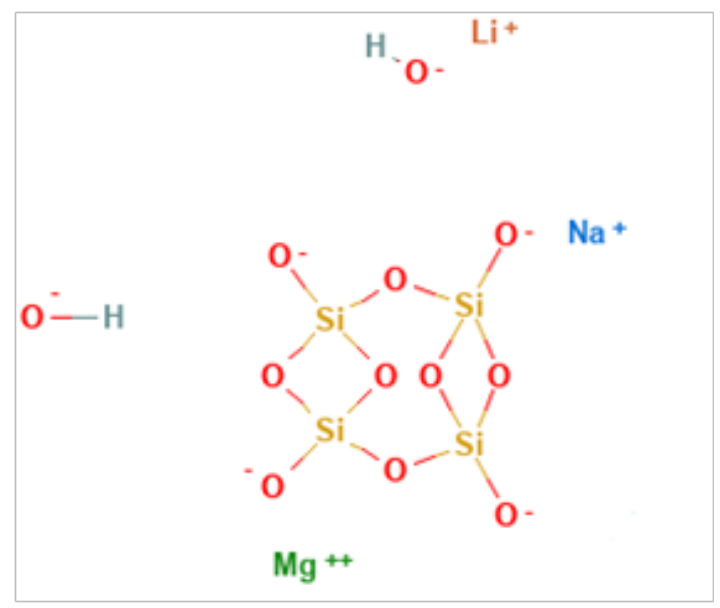

Figure 3 2-D structure of hectorite.
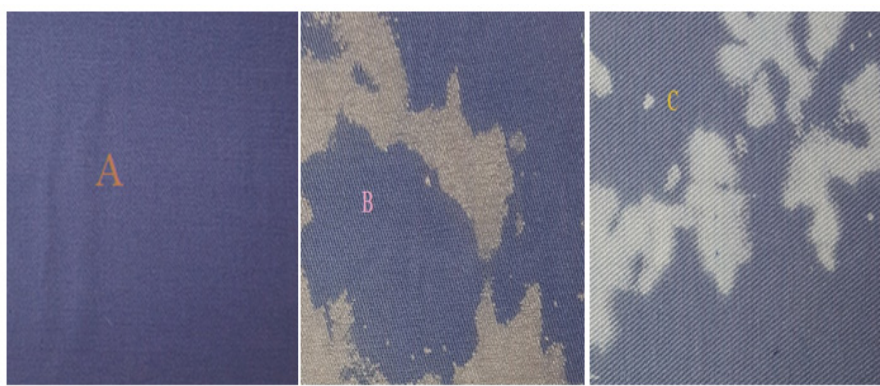

Figure 4 Cotton fabric dyed with Optisol Blue $C$ liq Sulphur Dye (A), Faded fabric with benzoyl peroxide (B), and Potassium Permanganate discharging agent (C).

\section{Materials and methods}

In this work, $100 \%$ cotton woven fabric, solubulised sulphur dyes (Optisul C Liquid) was used for dyeing and three types of oxidizing agents (potassium permanganate, benzoyl peroxide and sodium perborate) and thickener (hectorite clay) were used for printing purpose. 


\section{Dyeing procedure}

The dyeing was carried out in a semi-continuous method. At first pretreated cotton sample was collected. Then auxiliary mixing bath was prepared. The bath was filled with water upto $1 / 3$ of the total volume. Then Emagin DPR liquid $15 \mathrm{~g} / 1$ and Dirsol MJ Liquid $7 \mathrm{~g} / \mathrm{L}$ was added. Then the mixture was stirred for 10minute. The Optisul
Blue C liquid was added with $10 \%$ (owd) hydrogen peroxide (50\%) which acts as reduction inhibitor. The $\mathrm{pH}$ of the bath was adjusted at 5-5.5 with Opticid PSD. Finally, the mixture was stirred for $10 \mathrm{~min}$. Then the fabric was padded in a padding machine. The padding was carried out at room temperature. The padding pressure was adjusted at $0.1 \mathrm{KPa}$. and $70 \%$ pick-up was maintained. Then the fabric was cured at $140^{\circ} \mathrm{C}$ for $90 \mathrm{sec}$ (Figure 5).

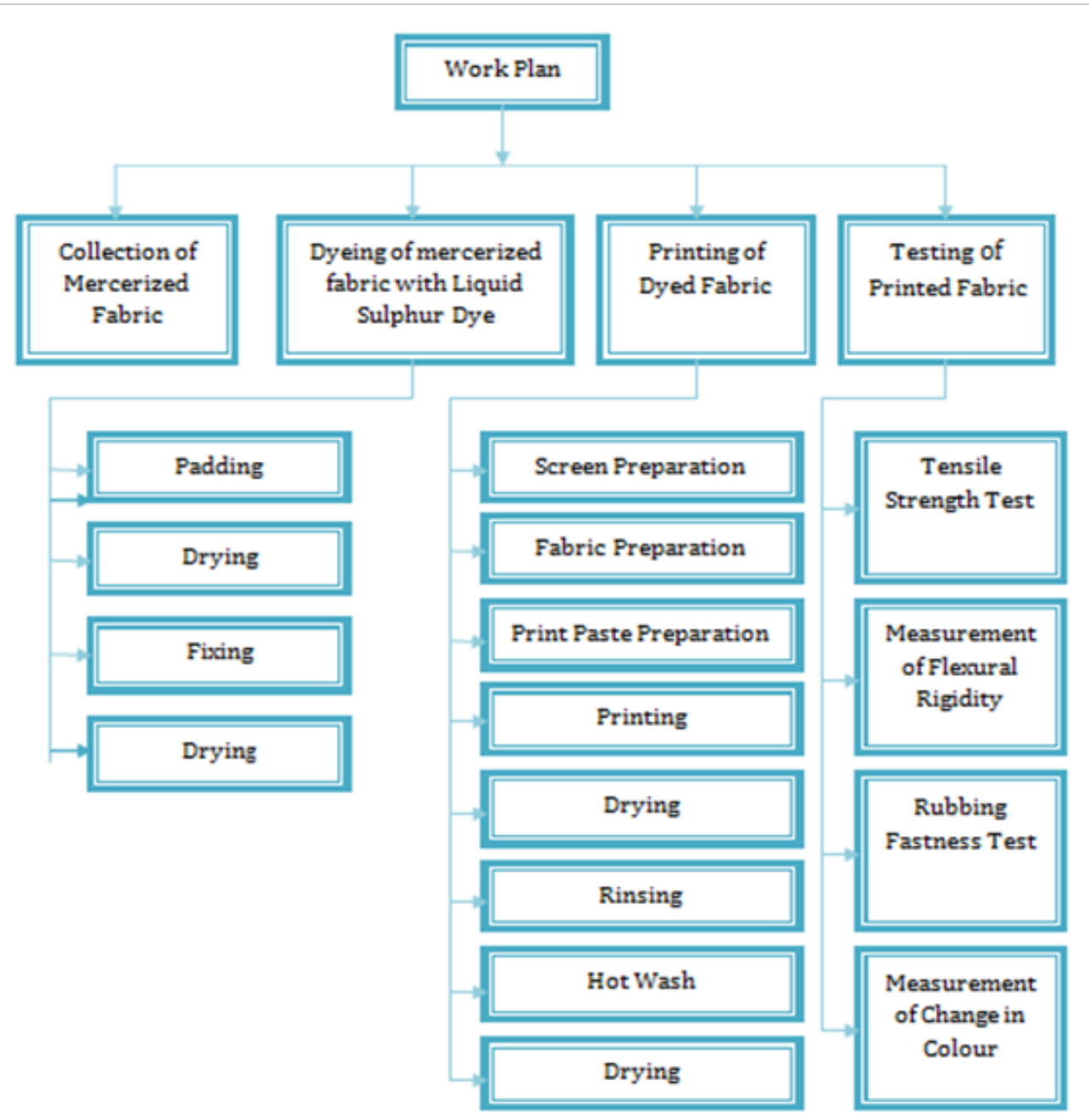

Figure 5 Work plan.

\section{Colour difference equations}

Quantitative colour differences using CIELAB: The values of $\mathrm{L}^{*}, \mathrm{a}^{*}$ and $\mathrm{b}^{*}$ for a given colour locate its position in the three dimensional CIELAB space. The colour difference between two similar non matching colours is the distance between the points for their respective coordinates in the CIE LAB colour space. This is calculated using the law of Pythagoras.

The colour difference $\Delta E_{a b}^{*}$ is:

$$
\Delta E_{a b}^{*}=\sqrt{\Delta L^{* 2}+P Q^{2}}=\sqrt{\Delta L^{* 2}+\Delta a^{* 2}+\Delta b^{* 2}}
$$

Where,

$$
\Delta L^{*}=\mathrm{L}_{\text {sam }}^{*}-L_{\text {std }}^{*} ; \Delta a^{*}=a_{\text {sam }}^{*}-a_{\text {std }}^{*} ; \Delta b^{*}=b_{\text {sam }}^{*}-b_{\text {std }}^{*}
$$

And where the subscripts 'sam' and 'std' refer to the sample and standard colour, respectively.

\section{Measurement of flexural rigidity}

Test method: BS 3356:1990: A rectangular strip of fabric (6inch $\times 1$ inch) is supported on horizontal platform in a direction perpendicular to one edge of the platform. The strip is extended in the direction of its length so that an increasing part overhangs and bends 
down under its own mass. When the tip of the specimen has reached a plan passing through the edge of the platform and inclined at an angle of $41.5^{\circ}$ below the horizontal. The overhanging length is equal to twice the bending length of the specimen. The flexural rigidity is obtained from the bending length and the mass per unit area of the fabric.

\section{Tensile strength test}

Test method: ASTMD 5035: A fabric test specimen $(18 \mathrm{~cm} \times 5 \mathrm{~cm})$ of specified dimensions is extended at a constant rate until it ruptures. The maximum force and the elongation at maximum force and if required, the force at rupture and the elongation at rupture are recorded. Grab and Strip technique are followed. The latter is followed in this project. For woven fabrics, each test specimen shall be cut with its length parallel to the warp or weft of the fabric and shall be sufficiently wide to allow the necessary fringes. Thread shall be removed in approximately equal numbers from each of the long edges of the cut strip until the width of the test specimen. The width of the fringes shall be such that during testing no longitudinal threads escape from the fringes.

\section{Colour fastness to rubbing}

Test method: ISO $105 \mathrm{X12:1993:} \mathrm{This} \mathrm{method} \mathrm{is} \mathrm{designed} \mathrm{to}$ determine the amount of colour transferred from the surface of coloured textile materials to other surfaces by rubbing. It is applicable to textiles made from all fibres in the form of yarn or fabric whether dyes, printed or otherwise coloured. Test procedures employing white test cloth squares, both dry and wet with water are given. As washing, dry cleaning, shrinkage, ironing, finishing etc. may affect the degree of colour transfer from a material; the test may be made before or after, or before and after any such treatment.

\section{Results and discussion}

\section{Measurement of change in colour}

The Figure 6 shows that both the concentration of the oxidizing agents and thickener affect the fading process. The higher the concentration of the oxidizing agents, the higher the effect of fading. Combination of benzoyl peroxide and hectorite gives the higher fading effect in all concentrations. The fading effects of potassium permanganate were slightly lower than that of benzoyl peroxide. The least fading effect was found in sodium perborate in all concentrations. Fading is achieved by the degradation of dye chromophore. The oxidizing agent oxidizes the dye chromophore to make it colorless. The carbonyl and sulphide groups of dye molecules perhaps get oxidized.

\section{Tensile strength test}

From the above Figure 7, it can be observed that the tensile strength of printed sample is slightly lower than the strength of dyed sample. The tensile strength has not fluctuated much regardless of the thickener and concentration of the oxidizing agent. The value of tensile strength of the most of printed samples are in the range of $950 \mathrm{~N}$ to $1200 \mathrm{~N}$ and $500 \mathrm{~N}-600 \mathrm{~N}$ in both warp and weft respectively, sufficient to meet consumers need. Type of oxidizing agent as well as concentration of oxidizing agent has significant effects on tensile strength of the fabric. This decrease in tensile strength might be due to a decrease in the degree of polymerization of cellulose resulting from formation of oxycellulose and hydrocellulose. Loss of tensile strength is not same for both colour of fabric. Different dyes may activate the oxidation and hydrolysis of cellulose to different extents. So the effect on tendering of the cellulosic fabric is likely to vary.

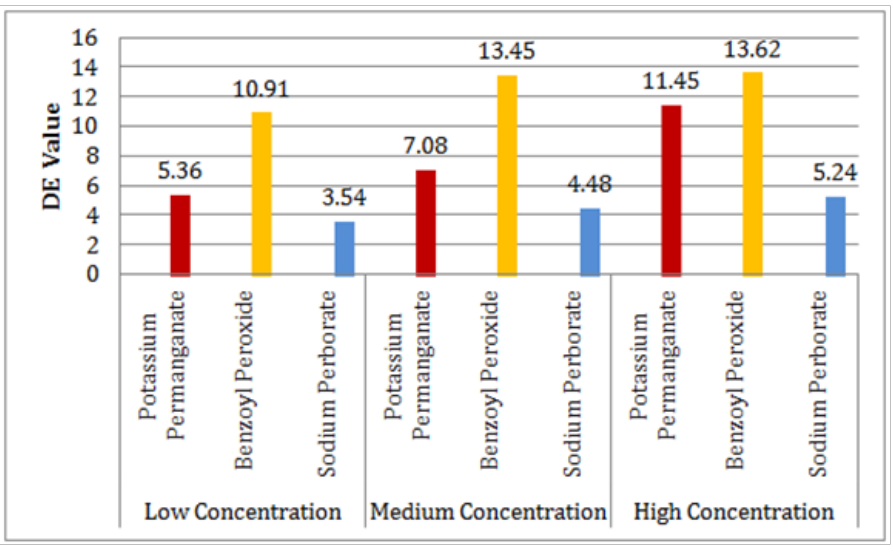

Figure 6 Colour difference between faded and unfaded portions of coloured sample.

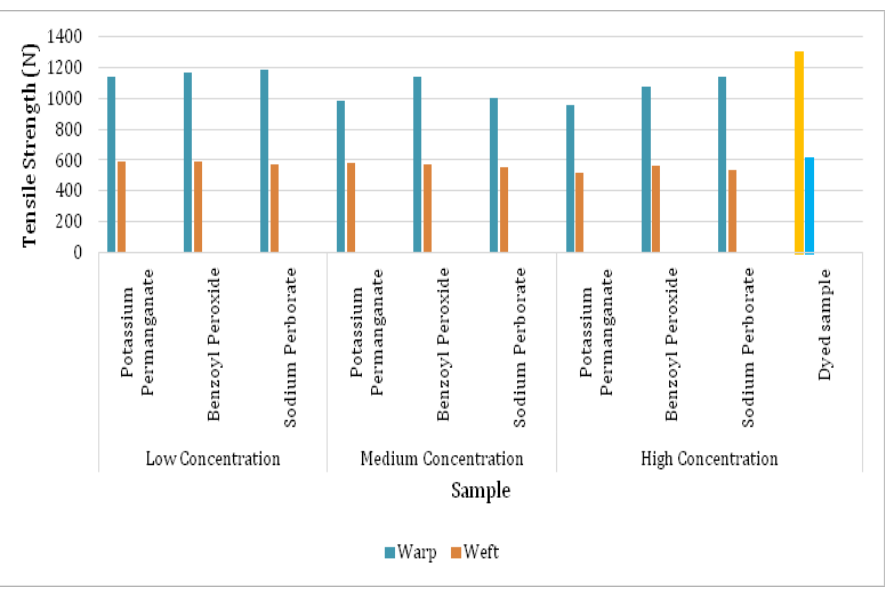

Figure 7 Tensile strength test results for coloured samples (warp \& weft).

\section{Colour fastness to rubbing}

From the Table 1, it can be commented that printed samples show less staining than the dyed sample in most of the cases. Combination of hectorite and all three oxidizing agents give good result in most cases. The staining of the samples ranged from moderate to very slight.

\section{Measurement of flexural rigidity}

From the above Figure 8, it is found that the flexural rigidity of printed fabric increased from that of unprinted dyed sample most of the time. When benzoyl peroxide is applied with hectorite, the value of flexural rigidity increases. From this it can be concluded that combination of benzoyl peroxide and hectorite shows greater stiffness compared to others. 
Table I Staining of crocking cloth for colour fastness to rubbing of coloured samples

\begin{tabular}{llllllllll}
\hline & \multicolumn{2}{l}{ Low concentration } & \multicolumn{2}{c}{ Medium concentration } & \multicolumn{2}{c}{ High concentration } \\
\cline { 2 - 9 } & PP & $\begin{array}{l}\text { Benzoyl } \\
\text { peroxide }\end{array}$ & $\begin{array}{l}\text { Sodium } \\
\text { perborate }\end{array}$ & PP & $\begin{array}{l}\text { Benzoyl } \\
\text { peroxide }\end{array}$ & $\begin{array}{l}\text { Sodium } \\
\text { perborate }\end{array}$ & PP & $\begin{array}{l}\text { Benzoyl } \\
\text { peroxide }\end{array}$ Sodium \\
perborate & sample \\
sectorite & 3.5 & 4 & 3.5 & 4.5 & 3.5 & 3.5 & 2.5 & 4 & 3 \\
\hline
\end{tabular}

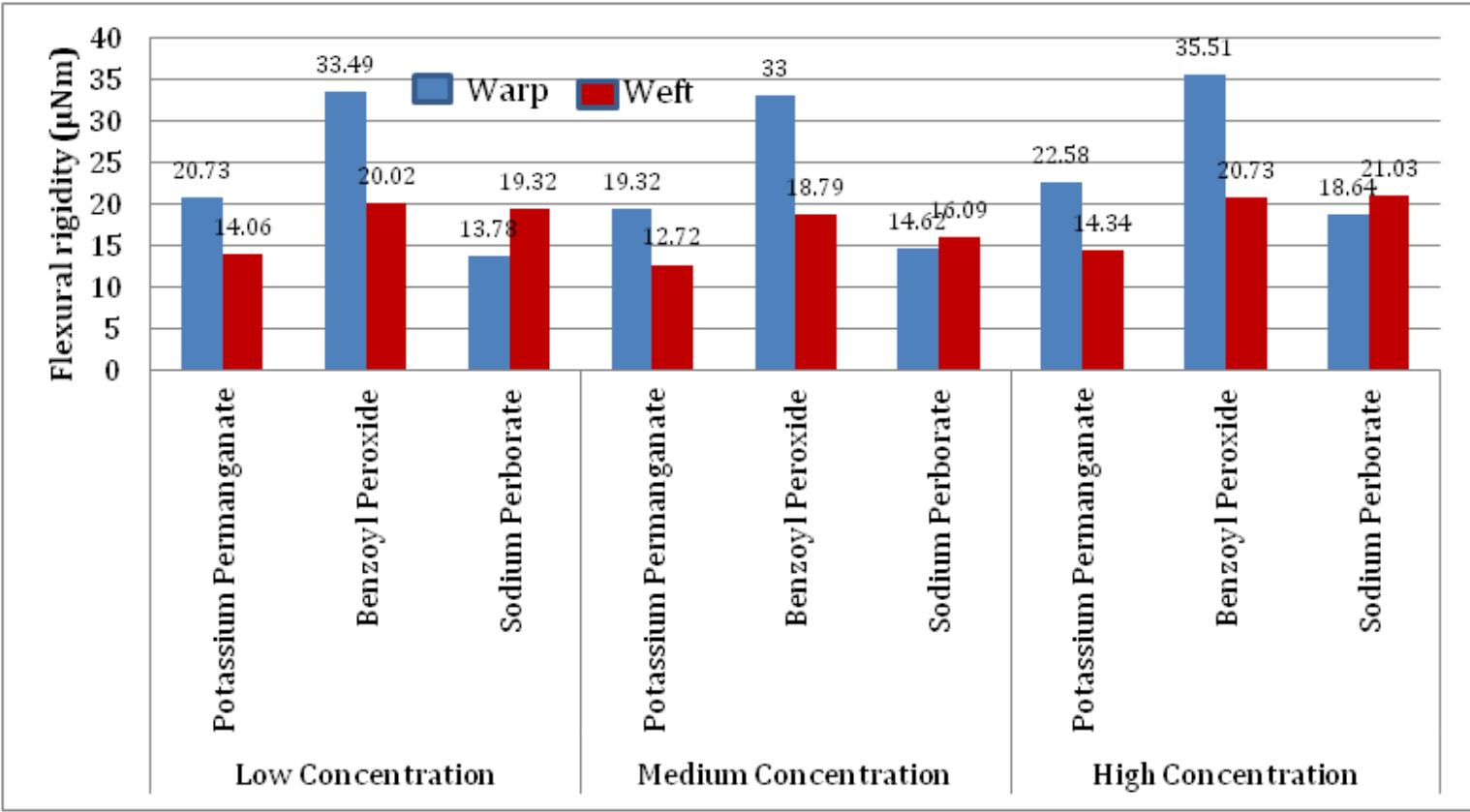

Figure 8 Flexural rigidity of test results for coloured samples (warp \& weft).

\section{Conclusion}

It is possible to create faded designs on cotton fabric by dyeing it in different colours with the new range of soluble sulphur dyes and subsequent discharge printing with oxidizing agents like potassium permanganate, benzoyl peroxide and sodium perborate and thickener like hectorite. The commercial importance of the process is similar to wash down effects, attractive discharge patterns on the indigo/ sulphur dyed denim fabrics could be produced which would enhance the aesthetic and commercial value. But the process widens the scope to produce faded effect on cotton fabric dyed in wide range of colours and definite patterns of our choice to meet the fashion demand.

\section{Acknowledgments}

None.

\section{Conflict of interest}

Author declares there is no conflict of interest in publishing the article.

\section{References}

1. Burkinshaw SM, Filarowski A. Physico-chemical aspects of textile coloration. Cambridge: John Wiley \& Sons; 2016. 648 p.

2. Chakraborty J. Fundamentals and Practices in Colouration of Textiles. Delhi: Woodhead Publishing India Pvt. Limited; 2014.

3. Maher RR, Wardman RH. The chemistry of textile fibres. London: Royal Society of Chemistry; 2015. 439 p.

4. Broadbent AD. Basic principles of textile coloration. Bradford: Society of Dyers and Colorists; 2001. 592 p.

5. Miles C, Leslie W. Textile Printing: Revised Second Edition. Bradford: Society of Dyers and Colourists; 2003. 352 p.

6. Guerra DL, Viana RR, Airoldi C. Use of raw and chemically modified hectorites as adsorbents for Th (IV), U (VI) and Eu (III) uptake from aqueous solutions. Desalination. 2010;260(1-3):161-71. 